\title{
Management of Traumatic Injuries of Road Traffic Accident Victims in the City of Ouagadougou at the University Hospital Trauma Emergency Department-Yalgado Ouédraogo
}

\author{
Alexandre S. Korsaga, Anatole J. I. Ouedraogo, Sayouba Tinto, Ives R. Kieno, \\ Mamoudou Sawadogo, M. Narcisse Dabire, Mohamed Tall, Namori Keita, Songahir C. Da \\ Yalgado Ouedraogo University Hospital Centre, Ouagadougou, Burkina Faso \\ Email: korsalexs@yahoo.fr, anatoleji2001@yahho.fr, swtinto@yahoo.fr, kienoivesroland@yahoo.fr, smamoudou@hotmail.com, \\ dabnar1@yahoo.fr, mohatall@yahoo.fr, borikeita@yahoo.fr, songahirda@gmail.com
}

How to cite this paper: Korsaga, A.S., Ouedraogo, A.J.I., Tinto, S., Kieno, I.R., Sawadogo, M., Dabire, M.N., Tall, M., Keita, N. and Da, S.C. (2019) Management of Traumatic Injuries of Road Traffic Accident Victims in the City of Ouagadougou at the University Hospital Trauma Emergency Department-Yalgado Ouédraogo. Open Journal of Orthopedics, 9, 212-223. https://doi.org/10.4236/ojo.2019.910022

Received: August 29, 2019

Accepted: October 19, 2019

Published: October 22, 2019

\section{Copyright $\odot 2019$ by author(s) and} Scientific Research Publishing Inc. This work is licensed under the Creative Commons Attribution International License (CC BY 4.0).

http://creativecommons.org/licenses/by/4.0/

\begin{abstract}
Purpose: To evaluate the management of traumatic injuries of accident victims in the city of Ouagadougou admitted to the trauma emergencies of the University Hospital of Ouagadougou. Material and Method: This was a descriptive prospective study of road traffic accident victims in the city of Ouagadougou on their arrival at trauma emergencies and on the 7th and 30th day after their discharge. A total of 991 patients were identified. The sex ratio was 1.94 with a male predominance. The average age of the patients was 31.5 years. The ratio of caregivers to patients on a 24 -hour shift was $7 / 47$, excluding patients hospitalized in the corridors. Results: We note that $81.94 \%$ of patients were transported by the fire brigade. The average time to first contact with a caregiver was 11 minutes. Upon admission, accident victims were accompanied in $84 \%$ of cases. The combination of paracetamol and néfopam was the most prescribed analgesic (50.2\%). The average time between admission and X-ray completion was 101 minutes. Benign skin lesions were the most frequent (48.82\%), followed by osteoarticular lesions of the limbs (fractures and dislocations). Ceftriaxone, and the combination of Amoxicillin + Clavulanic Acid, were the most prescribed antibiotics. Dressings and sutures (44.63\%) were the most commonly performed treatments, followed by orthopaedic restraints $(37.88 \%)$ and open fracture areas in $14.34 \%$. The average time between admission and orthopaedic and/or surgical treatment was 04 hours 25 minutes. Patients discharged against medical advice accounted for $10.80 \%$ of cases. In $95 \%$ of cases, patients were satisfied with their manage-
\end{abstract}


ment. Conclusion: The management of patients admitted to trauma emergencies is satisfactory, but difficulties remain in terms of delays in management. It is imperative to take into account certain factors involving both staff and working conditions in order to reduce the time required to provide care and improve user satisfaction in this emergency unit.

\section{Keywords}

Emergencies, Trauma, Treatment, Satisfaction

\section{Introduction}

Today, road traffic accidents are a major public health problem [1]. According to the World Health Organization's 2014 report, road traffic accidents killed an estimated 1.25 million people worldwide in 2013 and injured between 25 and 50 million people. These accidents were the leading cause of death among young people aged 15 to 29 worldwide in 2012; and the highest rate was observed in Africa (26.6 deaths per 100,000 inhabitants) where road accidents are the 5th leading cause of death. Surgical emergency departments, the usual place of admission for accident victims, often have malfunctions, particularly in Africa [2]. At the Yalgado Ouédraogo University Hospital (CHU-YO) in Ouagadougou, road traffic accidents are the second leading cause of death, after malaria [3]. Timeliness and quality of care are factors that influence morbidity and mortality. Unfortunately, very high attendance in a hospital does not ensure optimal patient care. Nowadays, the real indicator for evaluating the performance of a health centre is not only its technical platform but also its ability to meet patients' needs and expectations in a global way [4]. The evaluation of the quality of care is an approach that makes it possible to guarantee each patient diagnostic and therapeutic procedures capable of ensuring a better result, in accordance with the current state of medical science [5]. Hence, the objective of this study, which is to evaluate the management of accident victims admitted to our trauma reference centre.

\section{Materials and Method}

This was a descriptive prospective study conducted over a period of 06 months, from February 1, 2015 to July 31, 2015. The Orthopaedic-Traumatology Department of the CHU-YO, a national centre of last level of reference, served as the framework for the study. The collection sheets were developed in collaboration with the Centre de Recherche du Centre Hospitalier Universitaire de Montréal (CRCHUM), the Institut de Recherche pour le Développement (IRD) and the Centre National de Recherches Scientifiques et Technologiques (CNRST), as part of a project on accidentology in Ouagadougou. Data were collected at the arrival of the patients, then after their hospital stay (7th and 30th days). Data were collected on pre-established collection forms that included, for each pa- 
tient, socio-demographic, anatomical-clinical, radiological, therapeutic and satisfaction variables. After the patient left the trauma emergency unit on days 7 and 30, the information was collected by telephone calls. Included were all patients admitted for a road traffic accident in the city of Ouagadougou and who agreed to participate in the study. Accidents outside the city of Ouagadougou were excluded, as well as patients who died on arrival and those who refused to participate in the study. Informed consent of patients and/or their carers was obtained before the interviews. Confidentiality and medical confidentiality have been respected. Our sample included 991 patients. The sex ratio was 1.94 with a male predominance. The average age of the patients was 31.5 years. The average number of patients received per 24-hour shift during our study was 30 . The ratio of caregivers to patients on a 24-hour shift was 7/47 excluding patients hospitalized in the corridors.

The data entry was performed on a microcomputer equipped with Microsoft office 2007 software. Data analysis was done using Epi info 3.5.1 software; Excel, SPSS, and Sphinx.

\section{Results}

\section{The mode of admissions to trauma emergencies:}

The National Fire Brigade (BNSP) urgently transported $81.94 \%$ of the injured (Table 1).

\section{Waiting times:}

- The average waiting time was 11 minutes with extremes from one (1) minute to one (1) hour 14 minutes. We note that $97.2 \%$ of the injured had their first contact with a medical staff in less than 30 minutes after their admission. The results are presented in Table 2.

- Arrival times for accompanying persons

The average time of arrival of a companion was 07 minutes. We note that $84 \%$ of patients were accompanied by a parent when they were admitted to the emergency room.

Table 1. Distribution of patients by emergency arrival mode $(n=991)$.

\begin{tabular}{ccc}
\hline Admission method & Number & $\%$ \\
\hline National Fire Brigade & 812 & 81.94 \\
Brought by a relative/friend & 106 & 10.70 \\
Referred from a Medical Centre with Surgical Antenna & 49 & 4.95 \\
Coming alone & 15 & 1.51 \\
Brought in by the person responsible for the accident & 04 & 0.40 \\
Referred from a Health and Social Promotion Centre & 03 & 0.30 \\
Brought by an accompanying person & 01 & 0.10 \\
Referred by a private health centre & 01 & 0.10 \\
Total & 991 & 100 \\
\hline
\end{tabular}


Table 2. Distribution of patients by Wait times $(n=991)$.

\begin{tabular}{ccc}
\hline Waiting times & Number & $\%$ \\
\hline Less than 15 min & 941 & 95 \\
15 to 29 min & 22 & 2.2 \\
30 to 44 min & 8 & 0.8 \\
45 to 59 min & 3 & 0.3 \\
60 to 1 hour 15 min & 17 & 1.7 \\
Total & 991 & 100 \\
Arrival times of the accompanying person & 84 \\
Accompanied sick people who arrived (00 min) & 832 & 11 \\
1 to 30 min & 109 & 2.7 \\
31 min to 01 hour & 27 & 0.8 \\
1 hour to 1 h 30 min & 8 & 0.1 \\
1 hour 30 min à 2 hours & 1 & 0.1 \\
$>02$ hours & 1 & 1.3 \\
Unaccompanied sick & 13 & 100 \\
Total & 991 & \\
\hline
\end{tabular}

\section{Prescriptions for analgesics and/or anti-inflammatories:}

The combination of paracetamol and néfopam was prescribed for half of the injured (50.2\%), compared to $27.21 \%$ for paracetamol and tramadol. Table 3 reports the prescription of analgesics and/or anti-inflammatories in 849 patients.

The delays between admission, the prescription of radiographic examinations and their completion:

611 patients (61.65\%) were prescribed radiological images, while 380 patients $(38.3 \%)$ had no indication of an X-ray prescription. These delays were identified in 598 patients.

- The average time between admission and the prescription of radiographic examinations was 53 minutes.

- The average time between the prescription of radiographic examinations and their completion was 48 minutes (with extremes of 07 minutes and 02 hours 11 minutes).

Thus, the average time between admission and radiographs is 101 minutes (1 hour 41 minutes). The results of these delays are shown in Table 4.

The prescription of scan examinations:

Eighty-nine patients (09\%) were prescribed scans. The brain scan was performed in 80 patients. An average time of one (1) hour and 40 minutes was observed between the admission of patients and the prescription for a CT scan, with extremes of 15 minutes and 02 hours and 55 minutes. Figure 1 summarizes the time between admission and the prescription of a scan examination.

\section{The different types of lesions:}




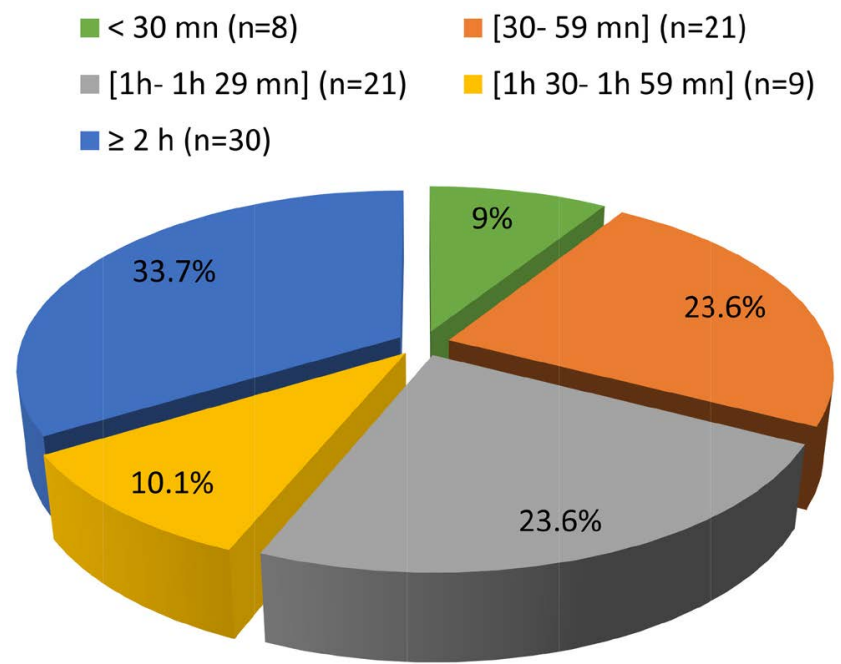

Figure 1. Time to complete scan examinations $(\mathrm{n}=89)$.

Table 3. Prescribed analgesics and anti-inflammatories $(\mathrm{n}=849)$.

\begin{tabular}{ccc}
\hline Prescribed analgesics/Anti-inflammatories & Number & $\%$ \\
\hline Paracetamol & 71 & 8.36 \\
Tramadol & 96 & 11.31 \\
Diclofenac & 5 & 0.6 \\
Kétoprofen & 1 & 0.1 \\
Paracetamol + Codeine & 5 & 0.6 \\
Paracetamol + Tramadol & 231 & 27.21 \\
Paracetamol + Nefopam & 426 & 50.2 \\
Paracetamol + Ibuprofen & 1 & 0.1 \\
Paracetamol + Diclofenac & 7 & 0.82 \\
Tramadol + Diclofenac & 5 & 0.6 \\
Tramadol + Ibuprofen & 1 & 0.1 \\
Total & 849 & 100 \\
\hline
\end{tabular}

Table 4. Distribution of patients by time of admission, prescription and emergency radiographs $(\mathrm{n}=598)$.

\begin{tabular}{cccccc}
\hline $\begin{array}{c}\text { Time limit for } \\
\text { radiographic prescription }\end{array}$ & Number & $\%$ & $\begin{array}{c}\text { Time limit for } \\
\text { radiographic production }\end{array}$ & Number & $\%$ \\
\hline$<30 \mathrm{mn}$ & 147 & 25 & $<30 \mathrm{mn}$ & 135 & 23 \\
{$[30 \mathrm{mn}-59 \mathrm{mn}]$} & 154 & 26 & {$[30 \mathrm{mn}-59 \mathrm{mn}]$} & 268 & 45 \\
{$[1 \mathrm{~h}-1 \mathrm{~h} 29 \mathrm{mn}]$} & 132 & 22 & {$[1 \mathrm{~h}-1 \mathrm{~h} 29 \mathrm{mn}]$} & 97 & 16 \\
{$[1 \mathrm{~h} 30-1 \mathrm{~h} \mathrm{59}]$} & 99 & 16 & {$[1 \mathrm{~h} 30-1 \mathrm{~h} \mathrm{59}]$} & 80 & 13 \\
$\geq 20 \mathrm{~h}$ & 66 & 11 & $\geq 20 \mathrm{~h}$ & 18 & 3 \\
Total & 598 & 100 & Total & 598 & 100 \\
\hline
\end{tabular}


A total of 2540 different types of injuries were diagnosed in the 991 injured. We note that $48.8 \%$ of the accident victims in our series had minor skin lesions, followed by osteo-articular lesions such as fractures and dislocations (28\%). The different types of injuries encountered are shown in Table 5.

\section{The prescription of biological examinations:}

No biological examination was prescribed in $78.61 \%$ of cases. On the other hand, 212 wounded (21.39\%) received a biological examination prescription, with a $93 \%$ completion rate (197 patients).

The prescription of care kits:

- Seven hundred and seventeen patients (72\%) received a prescription for kits for their management during their stay in trauma emergencies. A total of 709 patients (approximately 99\%) honoured the prescription of their kit, including 304 kits for local care. Table 6 shows the distribution of patients by type of medical kit prescribed.

- The average time between prescribing and obtaining the kits was 13 minutes with extremes of 02 minutes and 01 h 42 minutes. $76 \%$ or 540 patients obtained their kit in less than 15 minutes compared to 37 (5\%) who obtained it after more than one (01) hour of waiting.

Table 5. Distribution of the different types of lesions.

\begin{tabular}{ccc}
\hline Types of injuries & Number & $\%$ \\
\hline Minor skin lesions & 1240 & 48.82 \\
Osteo-articular lesions & 712 & 28.03 \\
Soft tissue injuries & 453 & 17.83 \\
Vascular lesions & 62 & 2.44 \\
Nerve damage & 41 & 1.61 \\
Dental injuries & 24 & 0.95 \\
Visceral lesions & 8 & 0.32 \\
TOTAL & $\mathbf{2 5 4 0}$ & 100
\end{tabular}

Table 6. Distribution of patients by type of medical kit prescribed $(n=717)$.

\begin{tabular}{ccc}
\hline Types of Kits & Number & $\%$ \\
No prescribed kit & 274 & 27.65 \\
Touching & 136 & 13.72 \\
Venous line & 157 & 15.85 \\
Touching + Venous tract & 130 & 13.12 \\
Venous route + Trimming & 92 & 9.28 \\
Touching + Venous track + Trimming & 37.73 \\
Venous route + upper limb plaster & 37 & 8.68 \\
Venous route + lower limb plaster & 86 & 7.97 \\
Total & 79 & 100 \\
\hline
\end{tabular}




\section{The prescription of medical treatments:}

A total of 536 patients had received a prescription for antibiotics, 531 (99\%) of whom filled their prescriptions. Ceftriaxone, and the combination of Amoxicillin + Clavulanic Acid were the most prescribed antibiotics. Table 7 summarizes the different types of antibiotics prescribed.

Among other medical treatments administered, serum and tetanus vaccine prescriptions, and anticoagulants were the most frequent. Figure 2 shows the distribution of patients according to the drug treatments received.

Orthopedic and/or surgical treatments:

Local care, in $44.62 \%$ of cases, accounted for the majority of emergency care through touching, sutures associated with simple dressings. Orthopedic care was followed in $37.91 \%$ and the trimming of open fractures in $14.34 \%$. Emergency osteosynthesis was performed in $3.12 \%$ of cases. These different types of processing are represented in Table 8.

Table 7. The different types of antibiotics prescribed $(n=531)$.

\begin{tabular}{ccc}
\hline Types of antibiotics & Number & $\%$ \\
\hline Fucidine ointment & 7 & 1.32 \\
Cefixime & 9 & 1.7 \\
Ceftriaxone + Amoxicillin/Clavulanic acid + Metronidazole & 56 & 10.55 \\
Ceftriaxone + Amoxicillin/Clavulanic acid + Ciprofloxacin & 29 & 5.46 \\
Ceftriaxone + Amoxicillin/Clavulanic acid & 99 & 18.64 \\
Ceftriaxone + Metronidazole & 60 & 11.3 \\
Ceftriaxone & 159 & 29.94 \\
Amoxicillin/Clavulanic acid & 101 & 19.02 \\
Ciprofloxacin & 11 & 2.07 \\
Total & 531 & 100 \\
\hline
\end{tabular}

Table 8. Distribution of patients by orthopedic/surgical treatment $(n=865)$.

\begin{tabular}{ccc}
\hline Types of Orthopaedic and/or Surgical Treatment & Number & $\%$ \\
\hline Clavicular ring & 25 & 2.89 \\
Removable knee splint & 17 & 1.96 \\
Plastered splint (cuff, Brachio-Anté-Brachio-Palmaire, Cruero-pedipal) & 125 & 14.45 \\
Necklace and corset & 28 & 3.24 \\
Ligament knee brace & 23 & 2.66 \\
Mayo clinic & 15 & 1.73 \\
Osteosynthesis & 27 & 3.12 \\
Simple dressing & 284 & 32.83 \\
Trimming of open fractures & 124 & 14.34 \\
Circular plaster & 95 & 10.98 \\
Wound suture & 102 & 11.8 \\
Total & $\mathbf{8 6 5}$ & 100 \\
\hline
\end{tabular}




\section{Delays between admission and therapeutic management:}

The average time between admission and orthopaedic and/or surgical treatment was 04 hours 25 minutes with extremes of 15 minutes and three (03) days. Figure 3 shows the time frames for therapeutic management.

Patient discharge patterns and satisfaction after hospital discharge:

- The number of injured persons authorized to return home was 833 (84\% of cases) compared to 158 injured (16\%) who were hospitalized. Thus, a total of 866 patients $(87.4 \%)$ had a stay of less than 24 hours in the Trauma Emergency Department.

- Regular discharges represented $74.37 \%$ of cases compared to $10.8 \%$ of discharges against medical advice. The majority of the injured were transferred to the hospitalization unit of the Orthopaedic-Traumatology Department (44.12\%) and the Maxillofacial Surgery Department (25.73\%). Table 9 summarizes the distribution of patients by discharge and transfer mode.

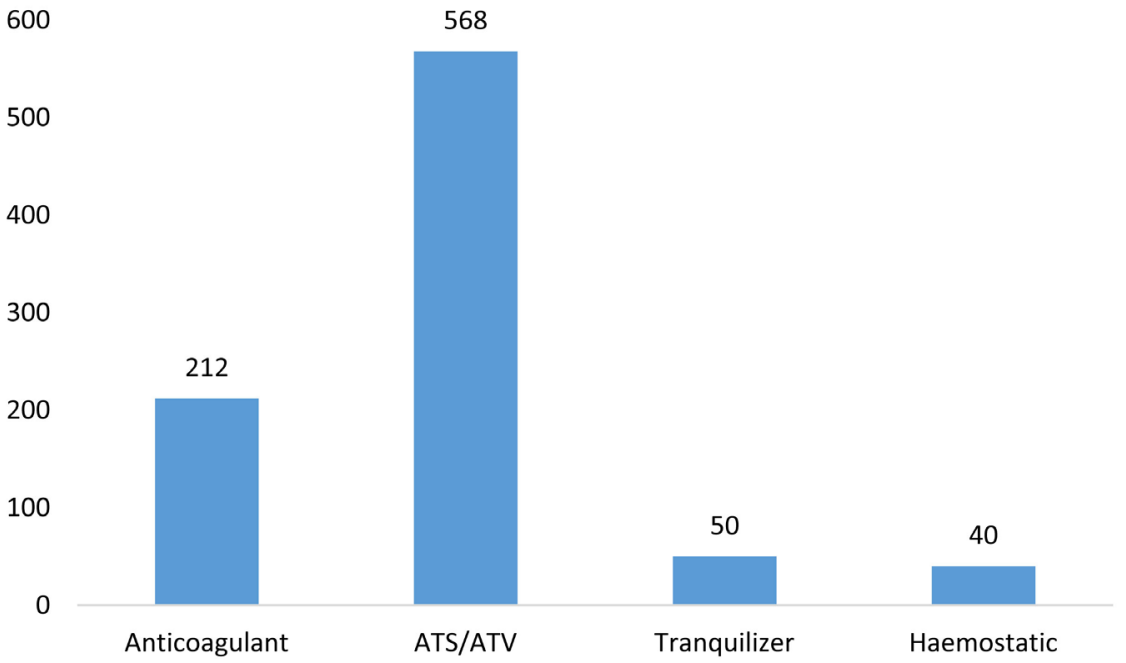

Figure 2. Distribution of injured persons by drug treatment.

Table 9. Distribution of patients by discharge and transfer mode.

\begin{tabular}{|c|c|c|c|c|c|}
\hline Patient discharge method & Number & $\%$ & Transfer services & Number & $\%$ \\
\hline Lost to follow-up & 11 & 1.11 & Orthopedics-Traumatology & 60 & 44.12 \\
\hline $\begin{array}{l}\text { Regular outings without } \\
\text { an attendant }\end{array}$ & 8 & 0.81 & Maxillofacial surgery & 35 & 25.73 \\
\hline Regular outings with support & 729 & 73.56 & Gynaecology/Obstetrics & 7 & 5.15 \\
\hline $\begin{array}{l}\text { Outings against medical advice } \\
\text { with accompanying person }\end{array}$ & 106 & 10.70 & Neurosurgery & 6 & 4.41 \\
\hline $\begin{array}{l}\text { Outings against medical advice } \\
\text { without accompanying person }\end{array}$ & 1 & 0.10 & Ophthalmology & 14 & 10.3 \\
\hline \multirow[t]{2}{*}{ Transferred } & 136 & 13.72 & ORL & 11 & 8.09 \\
\hline & & & Visceral emergencies & 3 & 2.2 \\
\hline Total & 991 & 100 & Total & 136 & 100 \\
\hline
\end{tabular}




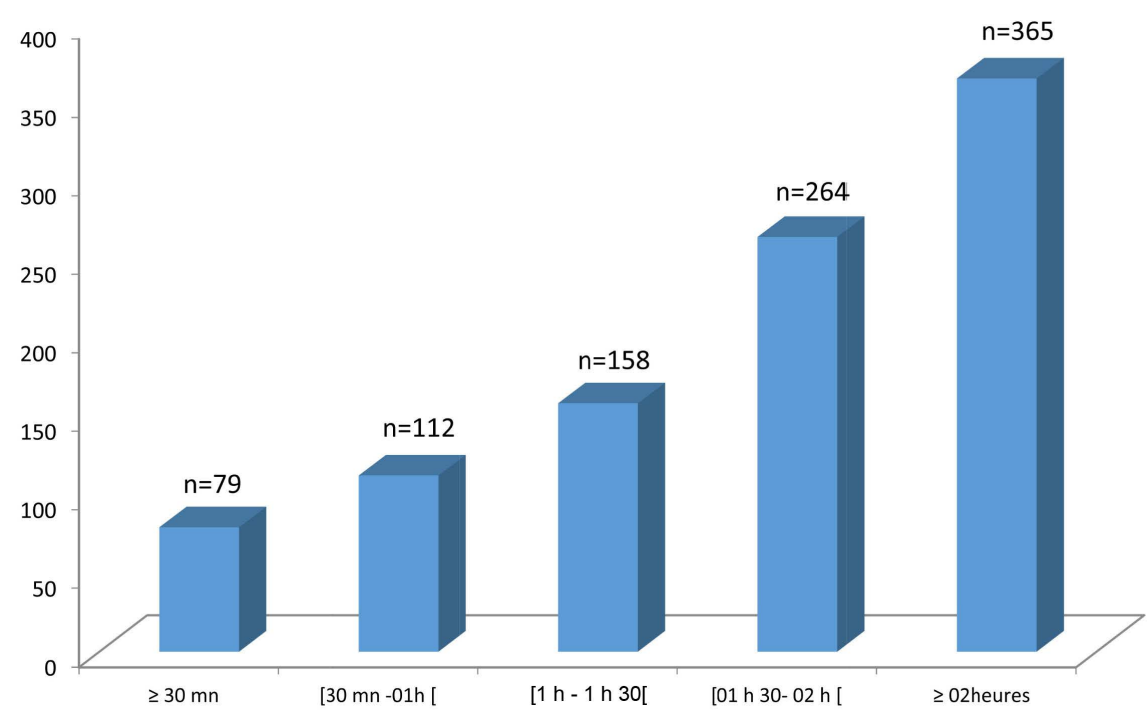

Figure 3. Distribution of patients by orthopedic/surgical treatment time $(n=978)$.

- Regarding the level of satisfaction of the injured, 987 of them gave their views: 650 patients (66\%) reported being very satisfied with their care, 288 (29\%) were satisfied and 49 (5\%) remained indifferent.

\section{Discussion}

Surgical emergencies in Africa are characterized by a chronic mismatch between patient needs, diagnosis and treatment [2].

Pain is the primary reason for emergency trauma consultation and its management reveals the level of patient satisfaction [4] [6]. Its impact must be systematically assessed from the first contact with the patient, but unfortunately this is not systematic in our emergency department [6]. In our working context, paracetamol is the most prescribed analgesic for emergency pain management [6], and it has been administered to $74.75 \%$ of our patients. Paracetamol and Nefopam were prescribed in $42.98 \%$ of patients, followed by paracetamol and Tramadol in $23.3 \%$ of cases. In addition, combinations of Tier 1 and Tier 2 analgesics are commonly used, and aim to seek a synergistic analgesic effect, more powerful to act on pain [6]. It is important to have a pain assessment and management protocol in trauma emergency management units [6].

Reception and waiting time before treatment are among the factors that negatively influence patient satisfaction [4] [7] [8]. The average time for orthopedic or surgical treatment was 04 hours 25 minutes in our study. Adamou et al. [4] suggested that the existence of management kits and especially the severity of the trauma make waiting time significantly shorter. Our patients waited on average 53 minutes to have a prescription for radiographic and biological examinations and one (1) hour and 40 minutes for a scanner prescription. Ricroch et al. [9] observed: 30 minutes for the start of first aid; in case of hospitalization $126 \mathrm{mi}$ nutes for the decision to hospitalize and 106 minutes in case of conventional radiography, 168 minutes in case of a biological procedure. The large number of 
people in our traumatological emergency justifies this difference in time. In short, these delays depend on the pathology, the type of establishment but also on the number of patients present in each structure, itself a function of the time of day [9]. Within our trauma emergency department, there is no medical imaging station or biology laboratory. These exams are sometimes even done outside the CHU-YO. In addition, radiography and laboratory examinations are no longer part of the non-prepaid care system. While this system was set up at the time to compensate for delays in treatment in the various surgical, gynaecological and medical emergency units. Abd Elaal et al. [5] in Sudan reported an average of 9.44 minutes of waiting time.

In our current study, $97.87 \%$ of patients performed the radiographic examinations prescribed for them. The low cost of standard radiography has enabled almost all of the injured to carry out this examination. The other patients who did not perform the radiographic examinations blamed the lack of financial resources. We noted an average time of 48 minutes between prescription and radiographic examinations. This relatively long delay could be explained by a number of factors: the comings and goings of accompagnants for invoicing between emergencies-the radiology service-the cash register, and the insufficient number of stretcher-bearers.

Ceftriaxone (29.94\%) was the most prescribed antibiotic, followed by Amoxicillin-Clavulanic Acid (19.02\%). Our results differ from those of Elkharrat et al. [1] who, in France, reported a predominance of penicillin prescription (57.2\%), followed by fluoroquinolones (20.8\%), and C3G (10.4\%). The lack of follow-up of protocols and recommendations regarding the choice of antibiotics is a reality in our department.

We noted a predominance of local care (simple dressings and sutures) in $32.83 \%$ of cases. At the time of our study, in the city of Ouagadougou, there were three medical centres with a surgical antenna and a university hospital located in the southern suburbs, which could accommodate moderate to severe traumatic emergencies; but the lack of sorting when the injured were picked up by the rescue workers of the National Brigade of Fire Brigades meant that even minor traumatic injuries were found in large numbers in our department. The average time of surgical treatment was 04 hours 25 minutes with extremes of 15 minutes and 03 days. Three factors can explain the slowness of surgical management in our emergency: the factor of the nursing staff, the quality of the technical platform and the solvency of the patient. Staff, although insufficient in number, are affected by a lack of willingness because they say they are sufficiently motivated financially, compared to the excessive workload. To this must be added frequent breakdowns both in the operating room (lack of oxygen and autoclave for sterilization), as well as in the medical imaging department (radiography and scanning). Consumable ruptures are also frequent: of labile pharmaceutical and blood products. In addition, there is a lack of financial resources for patients to pay for surgical prescriptions, which cost an average of 600 US dollars. The introduction of universal health insurance, which could help to reduce the time 
required to provide care, is still awaited in our country. Very often, the absence of a third party payer is equivalent to the absence of full coverage.

The average length of stay was 01 day 05 hours. The high frequency of minor trauma in our emergency department explains this result, as $83.85 \%$ of patients were discharged after treatment.

Patients discharged against medical advice (or under discharge) accounted for $10.8 \%$ of patients. Is the population that does not find satisfaction in our hospitals? What is certain, the influence of beliefs and the accessible cost of traditional medicine compared to modern medicine in Africa, still motivates a significant part of the population to use traditional medicine [10].

Satisfaction reflects an individual's judgment resulting from the transformation of objective findings into subjective judgments [8] [12]. Thus, it is seen as an emotional response, a value judgment of the subjective experience lived by the individual on the service he or she experienced on an initial baseline [12]. The evaluation of user satisfaction with public services has been widely used to improve the quality of service provided to users [4] [8] [12]. In our current study, $95 \%$ of our patients were satisfied, $66 \%$ of whom were very satisfied with their management. Compared to the literature, our rates are higher than those found by several authors [4] [8] [11]. Caregiver competence, courtesy and satisfactory reception are factors that positively influence patient satisfaction [7] [8]. This is reflected in the low rates of dissatisfied 5\% in our series and $4.4 \%$ in Nguyen's in Vietnam [13].

Although prospective, our study had some limitations, namely that:

- Some patients probably could not be collected;

- The subsequent lack of follow-up of patients after they left the trauma emergency unit (after D30) did not allow us to assess the evolution and satisfaction rate in the medium and long term.

\section{Conclusion}

The care of road traffic accident victims in the city of Ouagadougou admitted to trauma emergencies is satisfactory, but difficulties remain in terms of delays in care, due to the inadequacy and "lack of motivation" of the medical staff, the inadequacy of the technical platform and the solvency of the patients. It is imperative to take these various factors into account in order to reduce the time required to provide care and improve user satisfaction in this emergency unit.

\section{Conflicts of Interest}

The authors declare no conflicts of interest regarding the publication of this paper.

\section{References}

[1] Elkharrat, D., Brun-Ney, D. and Crdier, B. (2002) Antibiotic Prescriptions in 34 Emergency Services in France. Infectious Diseases, 29, 91-93. 
[2] Andreu, J.M. (2002) Surgical Emergencies in Africa (Does the Tropical Emergency Exist?). Med Trop, 62, 242-243.

[3] National Road Safety Office (ONASER) (2012) General Report of the National Forum on Road Safety. Ministry of Transport, Posts and Digital Economy, Ouagadougou, $18 \mathrm{p}$.

[4] Adamou, H., Amadou Magagi, I., Habou, O., Halidou, M., Karimou, S., Sani, R. and Abarchi, H. (2017) Patient Satisfaction, an Important Element in Overall Care: The Case of a Surgical Emergency Department in Niger. Le Mali médical, 32, 24-30.

[5] Abd Elaal, I.Y.A. (2006) The Waiting Time at Emergency Department at Khartoum State. Sudanese Journal of Public Health, 1, 122-126.

[6] Kabore, R.A.F., Ki, K.B., Traore, A.I., Bugouma, C.T.W., Damba, J., Bounkoungou, P.Z., Sanou, J. and Ouédraogo, N. (2014) Evaluation of the Knowledge and Practices of Ouagadougou Trauma Emergency Personnel on Pain Management. Assessment of Knowledge and Pain Management by Traumatological Emergencies Staff of the Teaching Hospital of Ouagadougou. Mali Medical, 29, 4.

[7] Revilla, A., Raffort, N., Plaisance, P. and Taboulet, P. (2019) Is Patient Satisfaction in the Emergency Room Comparable to That of Caregivers? Elsevier Masson SAS, Paris, S127.

[8] Attard, N., Vitasse, Y., Nouvellet, S., Kurtzemann, I., Pisapia, M., Galiez, F., et al. (2019) Adult EPS User Satisfaction Survey. Interests and Limitations of a Prospective Study. Elsevier Masson SAS, Paris, S127.

[9] Ricroch, L. and Vuagnat, A. (2015) Travel Time and Delays to the Emergency Room; Results of the National Survey. Journal of Epidemiology and Public Health, 63, 18-19. https://doi.org/10.1016/j.respe.2015.01.040

[10] Institut National de la Statistique et De La Démographie, Burkina Faso (2002) Opinion Survey of Users of Basic Public Services.

[11] Kasouati, J., Bouti, L., Zidouh, O., Abd El Hamid, Z., Boufaresse, A. and Mrabet, M. (2015) Evaluation of Patient Satisfaction with Pain Management in the Emergency Department of the Mohamed V Military Hospital of Instruction; Rabat Maroc. Revue D'épidémiologie et de Santé Publique, 63, 575. https://doi.org/10.1016/j.respe.2015.03.085

[12] Ragno, A. (2006) Evaluation of User Satisfaction with Outsourced Local Public Services; Orléans Laboratory for Management. Journal of Epidemiology and Public Health, 42.

[13] Nguyen, T., Emperor, F. and Briançon, S. (2002) Satisfaction of Patients Hospitalized at Ho Chi Minh City Hospital, Vietnam. Sante Publique, 14, 345-360. 